\title{
Study of how the synthesis conditions of circular optical scales fabricated using a CLWS-300 laser image generator affect their angular errors
}

\author{
D. U. Kruchinin, O. B. Yakovlev, ${ }^{\text {a) }}$ and M. P. Andronov \\ First President of Russia B. N. Yeltsin Ural Federal University, Ekaterinburg \\ (Submitted November 22, 2011) \\ Opticheskiǔ Zhurnal 79, 41-44 (July 2012)
}

This paper discusses the results of a study of how the synthesis conditions of circular optical scales fabricated using a laser image generator affect their angular errors. (C) 2012 Optical Society of America.

\section{INTRODUCTION}

The angular errors of dials fabricated by inverse photolithography ${ }^{1,2}$ are largely determined by the angular errors of the photomasks fabricated using the CLWS-300 laser image generator. ${ }^{3}$ It has been established that the angular errors of the circular optical scales synthesized by means of this generator are largely determined by the specific conditions under which they are synthesized. ${ }^{4}$ The dependence $F_{\text {ig }}(x)$ of the angular errors of a circular scale determined by the image generator is the sum of the systematic $F_{\operatorname{ig} 1}(x)$ and random $F_{\text {ig2 }}(x)$ dependences of the angular errors determined by the image generator, where $x$ is the angular position of the ruling. The cumulative error of position $F_{\text {ig }}(x)$ of the diameters of the axes of the rulings can reach more than $4^{\prime \prime}$.

In order to reduce the angular errors of circular scales fabricated using a laser image generator, it is expedient to determine the factors that can affect them. Such factors can include the method of fixing the chrome-plated substrate on the table of the generator spindle and the tilt angle and direction of this table.

The result of measuring the angular errors of a circular scale can be written as ${ }^{4}$

$$
F(x)=F_{m}(x)+F_{\text {ig }}(x)+F_{\text {sub }}(x),
$$

where $F_{m}(x)$ is the dependence of the angular errors introduced by the AS700 monitoring apparatus, while $F_{\text {sub }}(x)$ is the dependence of the angular errors introduced by the substrate.

The phase-statistical method makes it possible to separate $F_{m}(x)$ from $F_{\text {ig }}(x)+F_{\text {sub }}(x) .{ }^{5}$

\section{EXPERIMENTAL TECHNIQUE}

The CLWS-300 laser image generator was used to synthesize a circular scale $90 \mathrm{~mm}$ in diameter, consisting of 360 dark rulings $8 \mu \mathrm{m}$ wide, on the same substrate six times. The zero ruling is narrowed by $0.5 \mu \mathrm{m}$ on each side. The same polished substrate used in Ref. 4 was used for this investigation. With each deposition of the scale, the substrate was mounted on the table of the generator spindle in such a way that the position of the narrowed ruling on the substrate corresponded to the position of the zero ruling during the first deposition of the scale in Ref. 4, and this makes it possible to use the $F_{\text {sub }}(x)$ dependence obtained in that paper, determined by the substrate, to distinguish the $F_{\text {ig }}(x)$ dependence. The angular error of the scale on the AS700 apparatus was then determined in eighteen measurements, with the substrate rotated relative to the spindle axis of the apparatus by $20^{\circ}$. The position of the narrowed ruling was fixed from the characteristic single overshoot on the dependences of the angular errors of position of the ruling boundaries. The measured results were processed using the phase-statistical method. Studies were carried out for the angular errors of position of the diameters of the ruling axes.

When the first, third, and fifth recordings were made, the substrate was fixed on the table of the generator spindle by means of vacuum; when the second, fourth, and sixth recordings were made, it rested freely on the spindle table and its possible displacements were restricted by means of a plastic material. When the first and second recordings were made, the table of the generator spindle was placed horizontally (the tilt of the table did not exceed $0.5^{\prime}$ ); when the third, fourth, fifth, and sixth recordings were made, the table was tilted by about $3^{\prime}$. The angle between the tilt direction of the table of the generator spindle for the third and fourth recordings and that for the fifth and sixth recordings is about $120^{\circ}$, and this is caused by the design of the generator.

\section{EXPERIMENTAL RESULTS AND DISCUSSION}

Six $F_{\text {ig }}(x)+F_{\text {sub }}(x)$ dependences were obtained from these measurements by processing the results (Fig. 1). The cumulative angular errors of position of the diameters of the ruling axes for these dependences are shown in Table 1. The $F_{\text {ig }}(x)$ dependence was obtained by subtracting the earlier established $F_{\text {sub }}(x)$ dependence ${ }^{4}$ from their dependences obtained here (Fig. 2). The cumulative angular errors of position of the diameters of the ruling axes for these dependences are also shown in the table.

It can be asserted on the basis of these data that the angular errors of a circular optical scale fabricated by means of a CLWS-300 laser image generator are determined by attaching the substrate to the table of the spindle generator, using the angle and the direction of its tilt.

When the recordings are made on a freely resting substrate, the cumulative angular errors of position of the diameters of the ruling axes are fairly constant as the direction and tilt angle of the table of the spindle generator varies. Thus, the variations of the dependence of the angular errors in column 2 of Table 1 lie within the limits 1.21 to $1.49^{\prime \prime}$; in 
(a)

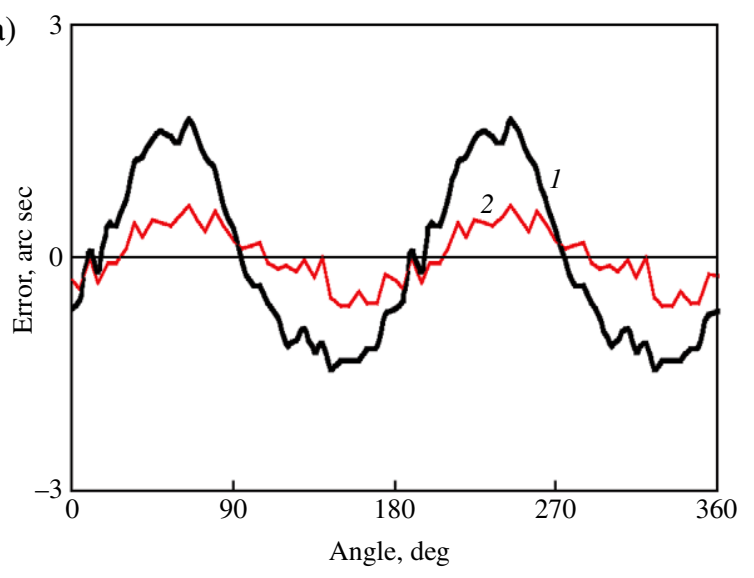

(b)

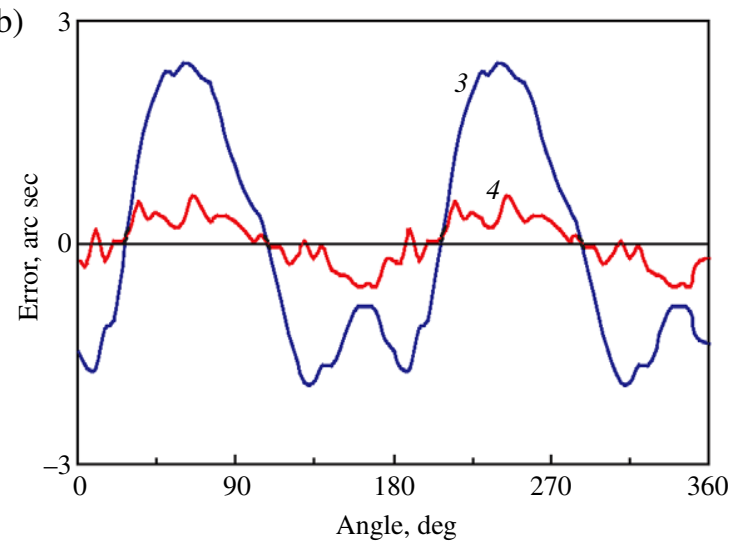

(c)

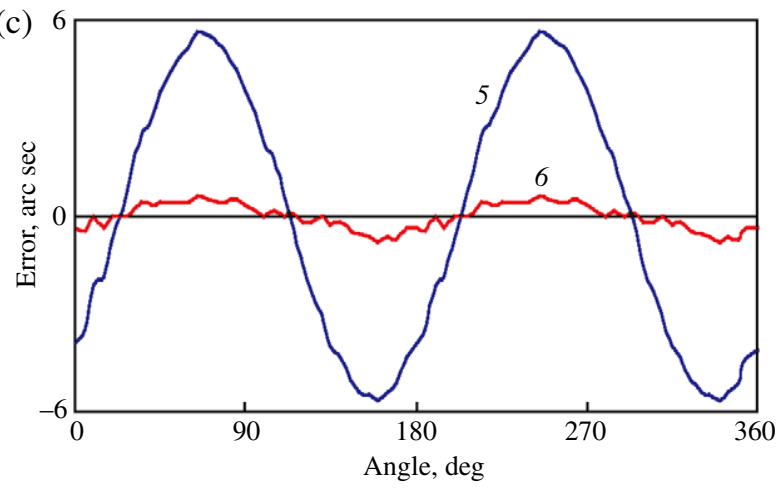

FIG. 1. (Color online) Dependences of angular errors $F_{\mathrm{ig}}(x)+F_{\mathrm{sub}}(x)$. The numbers on the curves correspond to the numbers of the recording.

TABLE 1. Cumulative errors of position of the diameters of the ruling axes.

\begin{tabular}{llll}
\hline \hline & \multicolumn{2}{c}{ Cumulative errors, arc sec } & \\
\cline { 2 - 3 } Recording No. & $F_{\text {ig }}(x)+F_{\text {sub }}(x)$ & $F_{\text {ig }}(x)$ & $\begin{array}{l}\text { Method of fixing the } \\
\text { substrate }\end{array}$ \\
\hline 1 & 3.19 & 2.29 & vacuum \\
2 & 1.29 & 1.41 & free \\
3 & 4.33 & 4.07 & vacuum \\
4 & 1.21 & 1.07 & free \\
5 & 11.30 & 10.60 & vacuum \\
6 & 1.49 & 1.26 & free \\
\hline \hline
\end{tabular}

column 3 from 1.07 to $1.41^{\prime \prime}$; in both columns from 1.07 to $1.49^{\prime \prime}$. The range of variations is less than $0.5^{\prime \prime}$. The maximum values of the cumulative errors (columns 2 and 3 of Table 1 ) for freely resting substrates do not exceed $1.5^{\prime \prime}$. These $F_{\mathrm{ig}}(x)$
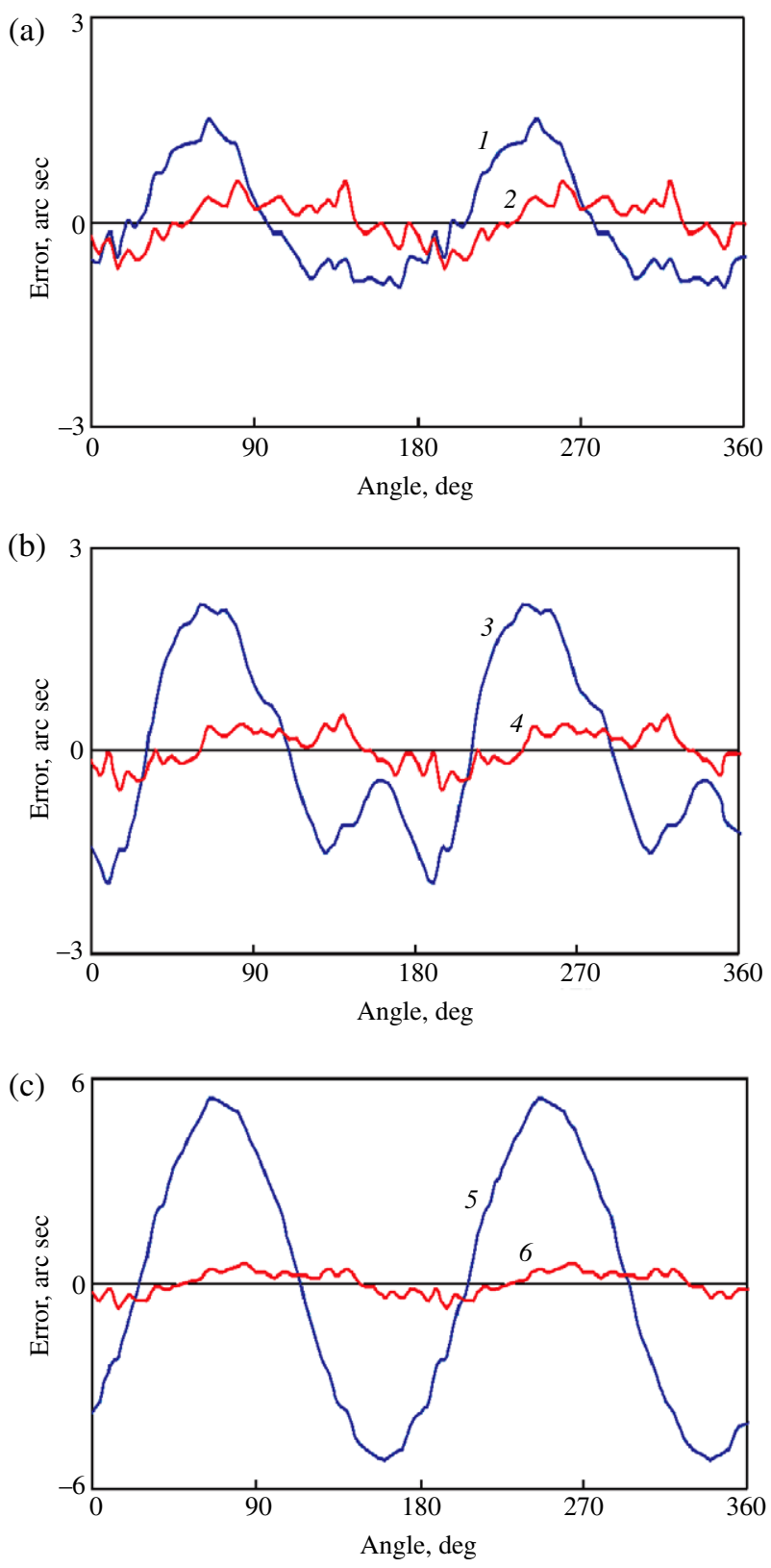

FIG. 2. (Color online) Dependences of the angular errors $F_{\mathrm{ig}}(x)$. The numbers on the curves correspond to the numbers of the recording.

dependences are mainly determined by the second harmonic (Fig. 2).

Vacuum fixation of the substrate significantly increases the angular errors of a circular scale. For a horizontal position of the table of the generator spindle (first recording), the cumulative angular error of position of the diameters of the ruling axes is increased by a factor of 1.6 (by comparison with the second recording). When the table is tilted during the recording, the cumulative errors increase by a factor of 7 or more. The direction in which the table is tilted during the recording can have a significant effect on the angular errors of the circular scale. The surface of the recording is apparently strongly distorted when there is vacuum fixation of the substrate, and this increases the angular errors.

It is thus established that the cumulative angular errors of position of the diameters of the ruling axes do not exceed 
$1.5^{\prime \prime}$ when a circular optical scale is recorded on a freely lying substrate.

The tilt of the table of the generator spindle (within the limits investigated here) and the direction in which it is tilted have no significant effect on the value of the cumulative error. There is no special meaning in this case in speaking of the separation of the dependence $F_{\text {ig }}(x)$ of the angular errors into systematic and random.

The use of vacuum fixation of the substrate during recording allows significant random dependences $F_{\text {ig2 }}(x)$ to appear in the angular errors of a circular optical scale.

\section{CONCLUSION}

As a result of these studies, the necessary conditions have been determined for synthesizing circular optical scales using a CLWS-300 laser image generator to make it possible to minimize their angular errors. The cumulative positioning errors of the diameters of the ruling axes of the synthesized scales do not exceed 1.5", and this is less than the values established by the manufacturer for this image generator $\left(2^{\prime \prime}\right){ }^{3}$
a)Email: OlegBJakovlev@yandex.ru

${ }^{1}$ D. U. Kruchinin, "Method of fabricating optical scales by inverse photolithography," Russian Patent No. 2370799 (2009).

${ }^{2}$ D. U. Kruchinin, "Photolithography in the production of circular optical scales at the Ural Optomechanical Factory," Opt. Zh. 75, No. 4, 92 (2008) [J. Opt. Technol. 75, 282 (2008)].

${ }^{3}$ Yu. F. Abramov, V. P. Kir'yanov, A. V. Kir'yanov, S. A. Kokarev, D. U. Kruchinin, Yu. V. Chuguĭ, and O. B. Yakovlev, "Modernizing the optical divider production of the Ural Optomechanical Factory on the basis of up-to-date laser-computer and photolithographic technologies," Opt. Zh. 73, No. 8, 61 (2006). [J. Opt. Technol. 73, 544 (2006)].

${ }^{4}$ D. U. Kruchinin and O. B. Yakovlev, "Study of the angular errors of circular optical scales fabricated by means of the CLWS-300 laser image generator,' Opt. Zh. 78, No. 6, 47 (2011). [J. Opt. Technol. 78, 383 (2011)].

${ }^{5}$ S. A. Bartik, S. E. Frizin, V. P. Kiryanov, A. V. Kiryanov, S. A. Kokarev, D. Y. Kruchinin, V. G. Nikitin, and O. B. Yakovlev, "Development of a technique for the determination of metrological parameters of technological system CLWS-300/C for synthesis of high-precision angle-measuring structures," in Tenth IMEKO TC7 International Symposium, Saint-Petersburg, 2004, pp. 316-320. 